\title{
Samsun ili kestane ağaçlarında görülen yaprakbiti, Lachnus roboris (Linnaeus, 1758) (Hemiptera: Aphididae) üzerine bir araştırma
}

\author{
A research of Lachnus roboris (Linnaeus, 1758) (Hemiptera: Aphididae) on \\ chestnut trees in Samsun
}

zzet AKÇA ${ }^{1 *}$

\section{Celal TUNCER $^{1} \quad$ Onur AKER $^{1}$}

\author{
Slam SARUHAN ${ }^{1}$
}

\begin{abstract}
Summary
In this study, Lachnus roboris Linnaeus (Hemiptera: Aphididae) on the samples of chestnut trees was recorded for the first time in the Ondokuz Mayis University Campus in Samsun and were examined morphologically in laboratory. Photographs of various body parts of apterous viviparous females of $L$. roboris were taken and morphological measurements of 40 apterous viviparous females and 120 eggs were also performed. In the study, egg length, body length, body width, antenna (left), (right), and corniculus of the pest were determined to be $1.70 \mathrm{~mm}$, $4.66 \mathrm{~mm}, 2.23 \mathrm{~mm}, 2.23 \mathrm{~mm}, 1.99 \mathrm{~mm}$ and $471.57 \mu \mathrm{m}$, respectively. In addition, PT/ BASE ratio of Ant.VI was found as 0.45 .
\end{abstract}

Key words: Lachnus roboris, aphid, Samsun, chestnut, morphology

\section{Özet}

Bu çalışmada, Samsun ili Ondokuz Mayıs Üniversitesi kampüsünde bulunan kestane ağaçlarında ilk defa görülen Lachnus roboris Linnaeus (Hemiptera: Aphididae) örnekleri laboratuvara getirilerek morfolojik olarak incelenmiştir. Kanatsız dişilerin değişik vücut parçalarının fotografları çekilmiş, 40 adet kanatsız dişi ve 120 adet yumurtanın morfolojik ölçümleri yapılmıştır. Yapılan ölçümler sonucunda; yumurta boyunun $1.70 \mathrm{~mm}$ olduğu, vücut uzunluğunun $4.66 \mathrm{~mm}$, vücut genişliğinin $2.23 \mathrm{~mm}$, Anten $2.23 \mathrm{~mm}$ (sol) ve $1.99 \mathrm{~mm}$ (sağ), rostum $1.59 \mathrm{~mm}$ ve Corniculus'un $471.57 \mu$ m olduğu belirlenmiştir. Ayrıca Ant VI da PT/ BASE oranı 0,45 olarak bulunmuştur.

Anahtar sözcükler: Lachnus roboris, yaprakbiti, Samsun, kestane, morfoloji

${ }^{1}$ Ondokuz Mayıs Üniversitesi, Ziraat Fakültesi, Bitki Koruma Bölümü, Samsun Sorumlu yazar (Correspondingauthor) e-mail: iakca@omu.edu.tr Alınış (Received): 10.12.2015

Kabul ediliş (Accepted): 04.01.2016 


\section{Giriş}

Türkiye, zengin bir flora, farklı coğrafi özellikler ve iklime sahip bölgeler içermektedir. Tüm bu özelliklere sahip olan ülkemizde, Aphid faunası ile ilgili çalışmalar sınırlıdır. Türkiye'de, 1980'li yıllara kadar 258 yaprakbiti türü belirlenmiş, 2014 yılına kadar bu sayı yaklaşık 500 olmuştur (Çanakçıoğlu,1975; Tuatay, 1991; Düzgüneş, 1982; Toros et al., 2003; Görür, 2002; Ölmez Bayhan et al., 2003; Aslan \& Uygun, 2005; Ölmez Bayhan et al., 2006; Özdemir et al., 2005; Remaudiere et al., 2006; Akyürek et al., 2011; Görür et al., 2012; Şenol et al., 2014; Özdemir \& Barjadze, 2015).

Çalışmanın konusu olan L. roboris türünün de bağlı olduğu Lachnus cinsine ait 20 türün değişik bitki türü üzerinde bulunduğundan bahsedilmektedir (Heie, 1995; Mifsud et al, 2009; Binazzi \& Kemaudiere, 2010; Kanturski, 2014) .

Görür et al. (2012), Türkiye faunasında Lachinini Tribe'sine bağlı 6 cins ve 11 tür olduğunu belirtmektedirler. Çalışmanın konusu, Lachnus roboris L. türü, Türkiye'de ilk defa 1922 yılında kayıtlara geçmiştir (Fahringer, 1922). Daha sonraki yıllarda, bu türün Türkiye'de; Ankara, Artvin, Diyarbakır, stanbul, Kahramanmaraş, Mardin, Samsun, Rize ve Trabzon illerinde varlığı değişik araştırmacılar tarafından rapor edilmiştir (Düzgüneş \& Tuatay, 1956; Bodenheimer \& Swirski, 1957; Çanakçıoğlu, 1967; Ölmez Bayhan et al., 2003; Aslan \& Uygun, 2005; Ülgentürk et al., 2009; Akyürek et al., 2012). Bu araştırmacılar, $L$. roboris türünü orman ve odunsu ağaç türlerinden olan çam, meşe ve ibreli ağaç türleri üzerinde bulmuşlardır. Akyürek et al. (2012), Samsun ilinde odunsu bitkilerde ve ayrıca kestane gal ve gövdesinde de $L$. roboris türüne rastladıklarını belirtmektedirler.

Lachnus roboris birçok odunsu ve ibreli ağaçlarda bulunan polifag bir türdür. Kestane ağaçları da bu türün konukçuları arasında bulunmaktadır (Blackman \& Eastop, 1994; Ülgentürk et al., 2009; Akyürek, 2013). Bu türün sinonim isimlerinden biri Lachnus castaneae 'dır (Akyürek, 2013). Ayrıca kestane ağaçlarında görülen yaprakbitleri ve bunların tanı anahtarları üzerine yapılan çalışmada, kestanede 30 yaprak biti türünden bahsedilmekte ve bunların içerisinde de $L$. roboris türü de bulunmaktadır (Blackman \& Eastop, 1994).

Günümüzde L. roboris türü üzerine yeterli bir çalışma bulunmamaktadır. Mevcut çalışmalar daha çok faunastik çalışmalar olup, morfolojik çalışma oldukça sınırlıdır. Son yıllarda Samsun ilinde Kestane ağaçlarında $L$. roboris türünün varlığı tarafımızdan tespit edilmiş olup, muhtemelen gelecek yıllarda popülasyonunda da belli bir artış olabileceği düşünülmektedir.

$\mathrm{Bu}$ çalışma ile Kestane ağaçları üzerinde bulunan L. roboris türüne ait bireyler incelenerek, tanınmasını kolaylaştırıcı bazı morfolojik özellikleri ve bazı vücut kısımlarının morfometrik ölçümleri yapılmıştır.

\section{Materyal ve Yöntem}

Çalışmanın ana materyalini, 2014 yılında Ondokuz Mayıs Üniversitesi Kampüsü kestane ağaçlarındaki $L$. roboris örnekleri oluşturmuştur. Kestane ağaçları üzerinde tespit edilen $L$. roboris örneklerinin öncelikle arazide fotoğrafları çekilmiştir. Ayrıca ağaç üzerinde toplanan örnekler, Ziraat Fakültesi Entomoloji Laboratuvarına getirilerek mikroskop (Olympus SZX16 Stereozoom Mikroskop) altında incelenmiş, 120 adet yumurta ve 40 adet kanatsız erginlerin bazı morfometrik ölçümleri alınmıştır.

\section{Araştırma Sonuçları ve Tartışma}

Lachnus roboris türü Hemiptera takımı, Aphididae familyası, Lachnus cinsine bağlı bir türdür. Sinonimleri; Aphis ilicicola, A. Iongipes, A. roboris, Dryaphiscerricola, D. licina, D. roborisnigra, Dryobiuscroaticus, Lachnus boerneri, L. castaneae, L. cerricola, L. croaticus, L. fasciatus, L. ilicicola, $L$. lepineyi, L. longipes, L. sachtlebeni, L. sessilis, Lygaeus hyalinatus, Schizodryobius boerneri (Blackman \& Eastop, 1994). Konukçuları: Castaneasativa, Picea sp. Pinus silvestris, Pinus sp., Quercus coccifera, Q. cerris, Q. ilex, Q. etraea, Q. pubescens, Q. robur, Q. suberi (Ülgentürk et al., 2009; Akyürek, 2013; Blackman \& Eastop, 1994) 


\section{Arazi gözlemleri}

Kestane ağaçları üzerinde yumurta, nimf ve kanatsız erginler görülmüştür (Şekil 1a). L. roboris yumurtalarını bir sıra halinde kestane dal ve sürgünlerinin üzerine bırakmaktadır. Yumurta bulunan kestane dallarında Coccinellid ve karıncalara da rastlanmıştır (Şekil 1b). L. roboris yumurtalarına odun karıncalarının (Formica rufa L.) saldırdığı bilinmektedir (Blackman \& Eastop, 1994).

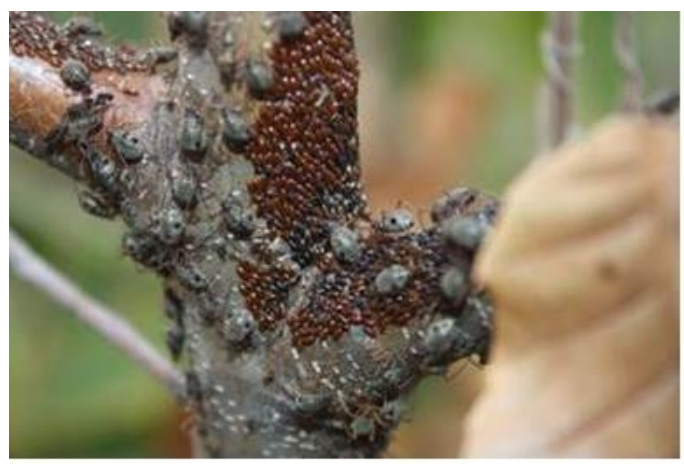

a

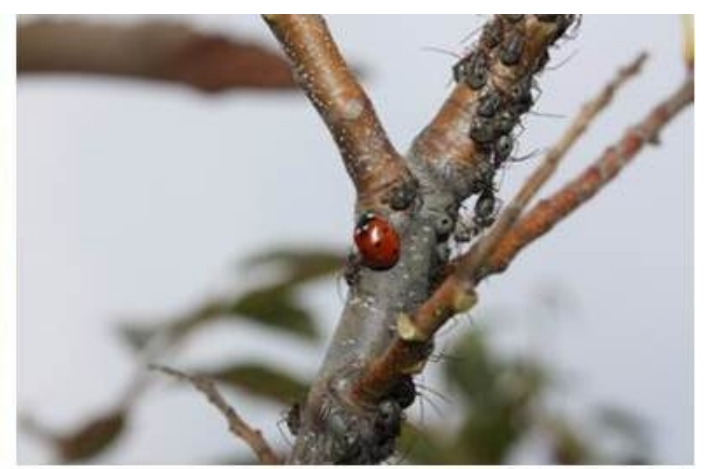

b

Şekil 1. Kestane dallarında Lachnus roboris yumurta ve kanatsız dişiler (a), Kanatsız dişi ve Coccinellid (b).

\section{Morfometrik özellikler}

Araziden getirilen yumurtaların ve kanatsız dişilerin bazı vücut kısımları mikroskop altında incelenmiş ve ölçümleri yapılmıştır. Ayrıca fotoğrafları da çekilmiştir (Şekil 2).

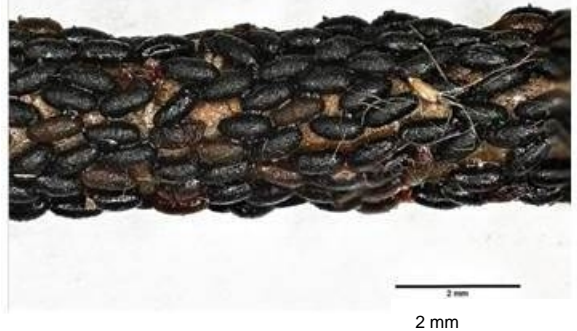

a

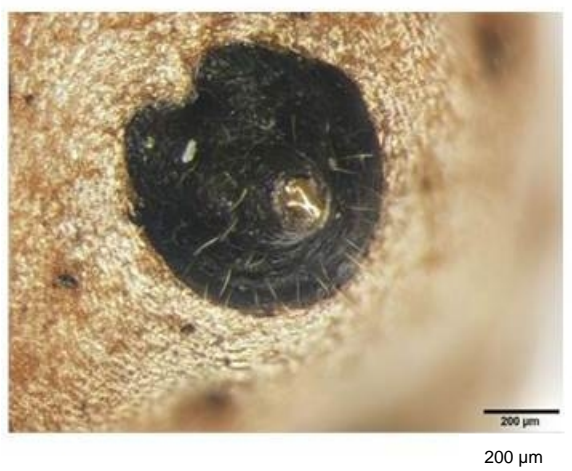

C

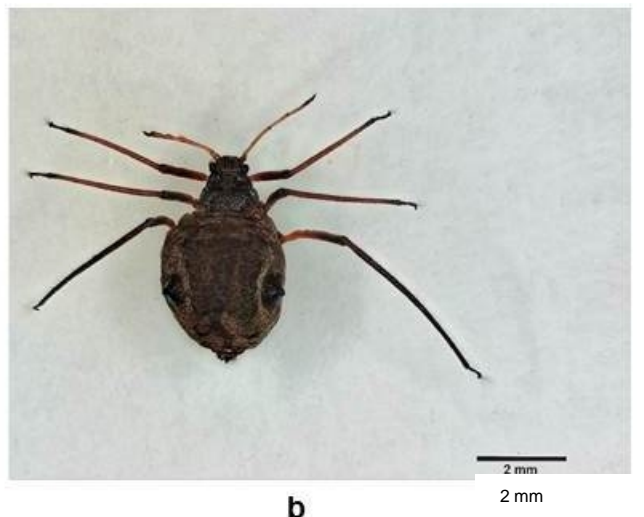

b

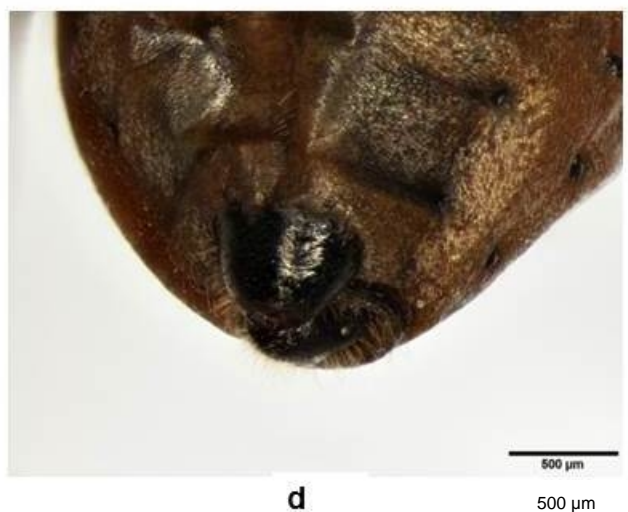

Şekil 2. Lachnus roboris türünün bazı vücut bölümleri; yumurta (a), Kanatsız dişi (b), Corniculus (c) ve Cauda (d). 
Laboratuvarda mikroskop altında yapılan incelemede; yumurtaların oval, Kanatsız dişilerin ise Coxa siyah, tibia ortasından itibaren uca doğru siyah, tarsusu siyah, trachanter ve femur kahverengi, femur-tibia birleştiği yer siyah, Thorax siyah, Cornicle siyah, Anten; 6. Segment siyah, diğerleri kahverengi, Cauda ve anal plakalar siyah, vücut deseni tüylü ve kahverengi, lateralde abdomende her segmentte siyah sağlı sollu yan plakalar var, ventralde her segmentte sağlı sollu laterale yakın siyah plakalar var, Rostrum özellikle uca doğru siyah olduğu gözlenmiştir (Şekil 2).

Vücut kısımları ile ilgili yapılan morfometrik ölçümler Çizelge 1'de gösterilmiştir. Yapılan ölçümlerde; yumurta boyunun $1.70 \mathrm{~mm}$ olduğu, vücut uzunluğunun $4.66 \mathrm{~mm}$, vücut genişliğinin $2.23 \mathrm{~mm}$, Anten $2.23 \mathrm{~mm}$ (sol) ve $1.99 \mathrm{~mm}$ (sağ), rostrum $1.59 \mathrm{~mm}$ ve Corniculus'un $471.57 \mu \mathrm{m}$ olduğu belirlenmiştir. Ayrıca Ant VI PT/Ant VI BASE oranı 0.45 olarak bulunmuştur. Özelikle L. roboris türünün diğer kestanelerdeki yaprak biti türlerinden ayıran özellikleri; Anteninin 6 segmentli olması, Corniculus'un geniş olması, en son anten segmentinin uç kısmının boyu (ANT PT)/ En son anten segmentinin taban kısmının boyuna (BASE) oranı 0,5 veya daha az olması, Vücut uzunluğu (BL) 'nun 2.5 mm'den fazla olmasıdır (Blackman \& Eastop, 1994).

Çizelge 1. Lachnus roboris türünün bazı vücut kısımlarının ölçümleri (mm)

\begin{tabular}{|c|c|c|}
\hline Karakter & Ortalama & Standart sapma $( \pm)$ \\
\hline Yumurta boyu (mm) & 1.70 & 0.01 \\
\hline Yumurta eni $(\mu \mathrm{m})$ & 742.17 & 3.13 \\
\hline Vücut uzunluğu (mm) & 4.66 & 0.36 \\
\hline Vücut genişliği (mm) & 2.78 & 0.17 \\
\hline Toplam anten (Sol) (mm) & 2.23 & 0.29 \\
\hline ANT I (sol) $(\mu \mathrm{m})$ & 124.50 & 23.54 \\
\hline ANT II $(\mathrm{sol})(\mu \mathrm{m})$ & 104.78 & 22.86 \\
\hline ANT III $(\mathrm{sol})(\mu \mathrm{m})$ & 898.27 & 74.50 \\
\hline ANTIV $(\mathrm{sol})(\mu \mathrm{m})$ & 452.01 & 53.12 \\
\hline ANTV (sol) $(\mu \mathrm{m})$ & 434.57 & 70.73 \\
\hline ANTVI (sol) PT $(\mu \mathrm{m})$ & 77.41 & 6.57 \\
\hline ANTVI (SOL) BASE $(\mu \mathrm{m})$ & 173.31 & 9.22 \\
\hline TOTAL ANT (Sağ) (mm) & 1.99 & 0.45 \\
\hline PT/BASE & 0.45 & 0.04 \\
\hline ANT I (sag) $(\mu \mathrm{m})$ & 124.14 & 22.06 \\
\hline ANT II $(\mathrm{sol})(\mu \mathrm{m})$ & 107.70 & 18.04 \\
\hline ANT III (sag) $(\mu \mathrm{m})$ & 861.05 & 95.32 \\
\hline ANTIV (sag) $(\mu \mathrm{m})$ & 456.61 & 66.72 \\
\hline ANTV $(\mathrm{sag})(\mu \mathrm{m})$ & 439.89 & 60.83 \\
\hline ANTVI -PT(SAĞ) $(\mu \mathrm{m})$ & 77.19 & 7.32 \\
\hline ANTVI-BASE(SAĞ) $(\mu \mathrm{m})$ & 171.05 & 10.78 \\
\hline PT/BASE sag & 0.45 & 0.04 \\
\hline ROSTRUM( mm) & 1.59 & 0.20 \\
\hline $\mathrm{R} I \mathrm{~V}+\mathrm{V}(\mu \mathrm{m})$ & 398.45 & 91.73 \\
\hline $\mathrm{HT} \mathrm{I}(\mu \mathrm{m})$ & 125.30 & 28.61 \\
\hline $\mathrm{HT} \|(\mu \mathrm{m})$ & 215.29 & 48.91 \\
\hline CORN CULUS $(\mu \mathrm{m})$ & 471.57 & 56.23 \\
\hline CAUDA (Ventral) $(\mu \mathrm{m})$ & 675.10 & 158.11 \\
\hline CAUDA (Dorsal) $(\mu \mathrm{m})$ & 279.21 & 94.24 \\
\hline
\end{tabular}




\section{Yararlanılan Kaynaklar}

Akyürek, B., 2013. Samsun li Aphididae (Hemiptera: Aphidoidea) Familyası Türlerinin Taksonomik yönden incelenmesi. OMU. Fen Bilimleri Enstitüsü, Doktora tezi, Samsun, 378s.

Akyürek, B., U. Zeybekoğlu \& G. Görür, 2011. Further contributions to theTurkey Aphid (Hemiptera: Aphidoidea) Fauna. J. Entomol. Res. Soc., 13(3): 101-106.

Akyürek, B., Ü. Zeybekoğlu, G. Görür \& M. Karavin, 2012. Samsun lindeki Odunsu Bitkiler Üzerinde Bulunan Yaprakbiti (Hemiptera: Aphididea) Türleri. 21. Ulusal Biyoloji Kongresi, 03-07 Eylül 2012, Ege Üniversitesi, zmir, 942s.

Aslan, M.M. \& N. Uygun, 2005. Aphids (Homoptera:Aphididae) of Kahramanmaraş Province, Turkey. Turk J. Zool. 29: 201-209.

Blackman, R.L. \& V.F. Eastop, 1994. Aphids on the world'strees: an identification and information guide. CAB International. $986 \mathrm{p}$.

Binazzi, A. \& G. Remaudière, 2010. The Palearctic - Oriental Fagaceae - feding species of Lachnus burmeister (Hemiptera; Aphididae, Lachninae). Redia XCIII: 45-73.

Bodenheimer, F.S. \& E. Swirski, 1957. The Aphidoidea of the Middle East. The Weigmann Science Press of Isreal, Jerusalem, $378 \mathrm{p}$.

Çanakçığlu, H., 1967. Türkiye'de Orman Ağaçlarında arız Olan Yaprakbitleri (Aphidoidea) Üzerine Araştırmalar. Tarım Bakanlığı, Orman Gn. Md. Yayınları Sıra No:466, Seri No: 22, VIII, 151 p.

Çanakçıoğlu, H., 1975. The Aphidoidea of Turkey. 1st Edition. University of Istanbul Forestry Faculty Press, $309 p$

Düzgüneş, Z. \& N. Tuatay, 1956. Turkiye Aphidleri. Ziraat Vekaleti, Ank. Zir. Enst. Md.Sayi: 4, 63 p.

Düzgüneş, Z, S. Toros, N. Kilinçer \& B. Kovancik, 1982. Parasites and predators of Aphidoidea species in Ankara province. Ministry of Agriculture and Rural Affairs, General Directorate of Protection and Control, 251s.

Fahringer, J., 1922. Eine Rhyncotenaus beuteaus der Turkei, Kleinasienund den Benachbarten Gebieten. Konowia, 1: $137-144$

Görür, G., 2002. New records forTurkish aphid fauna (Homoptera: Aphididae). Zool. Middle East, 25: 67-69.

Görür, G., H. Akyildirim, G. Olcabey \& B. Akyurek, 2012. The aphid fauna of Turkey: An updated checklist. - Arch. Biol. Sci., Belgrade, 64 (2): 675-692.

Heie, O.E., 1995. The Aphidoidea of Fennoscandia and Denmark VI. Aphidinae. Part 3 of Macrosiphini and Lachnidae. Fauna Entomologicas Candinavica, 31: 222 p.

Kanturski, M., K. Wieczorek \& L. Junkiert, 2014. A newoak-feedingspecies of Lachnus burmeister and some remarks on the taxonomic status of L. chosoni Szelegiewicz (Hemiptera, Aphididae, Lachninae). Dtsch. Entomol. Z., 61(1): 77-83

Mifsud, D., N.P. Hidalgo \& S. Barbagallo, 2009. Aphids (Hemiptera: Aphidoidea) associated with native trees in Malta (Central Mediterranean). Bulletin of the Entomological Society of Malta, 2: 81-93.

Ölmez Bayhan, S., M.R. Ulusoy \& E. Bayhan, 2003. Determination of Aphididae (Homoptera) fauna of Diyarbakir Province of Turkey. Turk. Entomol. Derg., 2003, 27(4) : 253-268.

Ölmez Bayhan, S., M.R. Ulusoy \& E. Bayhan, 2006. Aphids and their predators in Malatya region and around, Turkey. Journal of Biological Sciences, 6(5): 954-957.

Özdemir, I., G. Remaudière, S. Toros, \& N. Kılınçer, 2005. New aphid records fromTurkey including the description of a new Lachnus species (Hemiptera: Aphididae). Rev. Fr. Entomol., 27(3): 97-102.

Özdemir, I. \& S. Barjadze, 2015. Some new records of aphid species (Hemiptera: Aphididae) from the Middle East and the Caucasus. Turk J Zool., 39: 1-3.

Remaudiere, G., S. Toros \& I. Ozdemir, 2006. New contribution to the aphid fauna of Turkey [Hemiptera, Aphidoidea]. Rev. Fr. Entomol,, 28(2): 75-96.

Şenol, Ö., H. Akyıldırım, G. Görür \& E. Demirtaş, 2014. New records for the aphid fauna (Hemiptera: Aphidoidea) of Turkey. Acta Zool Bulgar, 66: 133-136. 
Toros, S., I. Özdemir \& H. Çanakçıoğlu, 2003. The Betula aphids of Turkey. J. Pest. Sci. ,76: 173-175.

Tuatay, N., 1991. Türkiye yaprakbitleri (Homoptera: Aphididae). Aphidinae: Macrosiphini (III. Kısım). - Bull. Plant. Prot, 31: 3-18.

Ülgentürk, S., I. Özdemir, Ö. Sahin, M. Mustu \& C. Erbaş, 2009. Ankara Kent Orman ve Parkları breli Ağaçlarında Bulunan Yaprakbiti (Homoptera:Aphidoidea) ve Kabuklubit (Homoptera: Coccoidea) Türleri, Acanthomytilus cedricola Balachowski et Alkan ve Leucaspis pusilla Low.(Homoptera: Diaspididae) Biyo-Ekolojileri. Ankara Üniversitesi Bilimsel Araştırma Projeleri Kesin Sonuç Raporu. 112s. 